\title{
Mass hierarchy from recoiling D-branes
}

\author{
G. K. Leontaris \\ Theoretical Physics Division, Ioannina University, GR-45110 Ioannina, Greece \\ and CERN, Theory Division, 1211 Geneva 23, Switzerland \\ N. E. Mavromatos \\ Theoretical Physics Group, Department of Physics, King's College London, Strand, London WC2R 2LS, United Kingdom
}

(Received 5 January 2000; published 4 May 2000)

\begin{abstract}
Using conformal field theory methods we construct a metric that describes the distortion of space-time surrounding a $\mathrm{D}$ (irichlet)-brane (solitonic) defect after being struck by another D-brane. By viewing our four-dimensional universe as such a struck brane, embedded in a five-dimensional space-time, we argue on the appearance of a band of massive Kaluza-Klein excitations for the bulk graviton which is localized in a region of the fifth dimension determined by the inverse size of the band. The band incorporates the massless mode (ordinary graviton) and its thickness is determined essentially by the width of the Gaussian distribution describing the (target-space) quantum fluctuations of the intersecting-brane configuration.
\end{abstract}

PACS number(s): 04.50.+h, 11.10.Kk, 98.80.Cq

\section{INTRODUCTION}

Considerable scientific interest has been concentrated recently on the revival and extension of the rather old idea that space-time is actually $4+n$ dimensional, with our fourdimensional world being a membrane (Dirichlet brane [1,2]) of some string theory living in a $(4+n)$ dimensional bulk space-time [3-8].

In some of these models, the extra (bulk) dimensions are taken to be relatively large, compared to the traditional Planck scale, implying, for instance, a bulk gravitational scale at the range of a few $\mathrm{TeV}$ [5-7]. Considerable effort has been devoted to a discussion of possible phenomenological consequences of these scenarios in immediate-future accelerators such as the CERN Large Hadron Collider (LHC).

In the case of extra compact dimensions, which is the one assumed in Refs. [5-7], there are induced modifications of the four-dimensional Newton's law, which may become phenomenologically important for $\mathrm{TeV}$ scale gravity $[9,10]$. Notice that such modifications are distinct from earlier modifications proposed in the context of supergravity [11]. It is straightforward to check that at least two extra dimensions are needed in order to avoid contradiction with the known laws of gravity at large (solar) distances. On the other hand, for $n=2$, astrophysical considerations [12] imply a scale $M \geqslant 10 \mathrm{TeV}$ which marginally solves the hierarchy problem. For larger $n$ there are less restrictions; however, it has been argued that in this approach the hierarchy problem essentially is reformulated in terms of another parameter which is now the compactification volume.

In the above scenario, the experimental success [13] of the inverse-square law of Newton seemed to imply precisely four noncompact dimensions only. More recently, however, the work of Ref. [8] has demonstrated that the situation is completely different in cases where the higher-dimensional metric was not factorizable [14]: namely; the case where there is a warp factor in front of the four-dimensional metric which depends on the coordinates of the bulk extra dimensions. According to this approach, our universe is a static flat domain wall which, in the simplest case of five dimensions, separates two regions of five-dimensional anti-de Sitter (AdS) space-time. In its simplest version [8], the scenario is realized by introducing a positive energy brane at the origin and a negative energy brane at distance $z$ where our world is located and where the graviton amplitude is exponentially suppressed. Modifications to the above picture with positive energy branes allowing also the possibility of infinite extra dimensions, multibrane solutions, and supergravity embedded versions were considered in the literature [15-22]. Thus, it is worth noticing that the bulk dimensions are not necessarily compact. The rather important point of Ref. [8], however, was the demonstration of the localization of the bulk gravitational fluctuations on the three-dimensional brane, which plays the role of our world. This localization property was demonstrated by mapping the problem of the dynamics of these fluctuations into a one-dimensional Schrödinger eigenvalue problem.

A characteristic feature of such models was the presence of a massless mode for the graviton (in agreement with Lorentz covariance on the brane) together with a continuum of massive Kaluza-Klein (KK) states on the four-dimensional world. These KK modes have different properties as compared with the factorizable case. The presence of such KK states leads to corrections of the four-dimensional Newton's law; such corrections, however, are suppressed by quadratic powers of the inverse Planck mass scale, and hence are unobservable for all practical purposes. In some variants of the model [23] one considers a periodic lattice of three branes, which generates bands in the Kaluza-Klein spectrum, separated from the massless graviton mode by a gap.

As a result of the above localization, a solution to the mass hierarchy emerges in the sense that the weak scale is generated from a large scale of the order of Planck mass through an exponential hierarchy, induced by the presence of the warp factor in the metric of the four-dimensional world.

The above models are very attractive, and indeed may offer a viable solution to the hierarchy problem. However, we find it rather restrictive that the discussions so far were 
concetrated only on static brane configurations without including dynamics.

Indeed, it is known $[24,25]$ that when one considers scattering of strings (or branes) off a D-brane, there is a nontrivial recoil of the latter which distorts the surrounding space-time [26], implying a sort of back reaction. Such a back reaction curves the space-time around the stringy defect in an nontrivial way. What we shall argue in this article is that, as a result of such a back reaction, one can obtain a different sort of mass hierarchy from that of Ref. [8], though the concept of an induced nonfactorizable bulk metric also appears here.

The recoil problem is treated at present perturbatively for heavy branes, within the context of a world-sheet logarithmic conformal field theory $[24,25,27]$. What we shall do in this work is to construct explicitly the space-time deformation due to the recoil of a 4-brane, viewed as our Euclideanized four-dimensional space-time embedded in a higher (five-) dimensional bulk space-time, after being struck by another brane. We shall demonstrate the localization of a thin band of KK massive bulk graviton modes (including the massless one) on our four-dimensional world, with thickness determined by a weak supersymmetry breaking scale $\alpha$ due to recoil [28]. We shall also demonstrate the formation of a horizon at distances given by the inverse of the thickness of the band of the localized KK modes. On this horizon there is localization of the rest of the massive KK modes, with masses higher than $\sim \alpha$. We shall also demonstrate that in this scenario the induced modifications of the fourdimensional Newton's law are suppressed by powers of $\alpha / M_{s}^{2}$, where $M_{s}$ is the string scale which in our case may be taken to be close to the Planck scale $M_{s} \sim 10^{18} \mathrm{GeV}$. Hence, such corrections are essentially unobservable for $\alpha \sim$ $\mathrm{TeV}$, which is the case dictated by the gauge hierarchy in our universe, given that $\alpha$ is the scale of the induced supersymmetry breaking on the 4-brane.

The structure of the article is the following. In Sec. II we present the salient features of the world-sheet approach to the D-brane struck by another D-brane or string. In Sec. III, we construct the space-time deformation due to the recoil effects and show that a nonfactorizable five-dimensional metric arises. We show the existence of a horizon located at a distance $z=1 / \alpha$ and discuss analytic continuation beyond the horizon. In Sec. IV we show that this metric is a solution to the Einstein equations describing an AdS universe with negative bulk-cosmological constant which vanishes at $z$ $=0$. We further show in the same section that the linearized Einstein equation leads to a Schrödinger-type equation with attractive potential for graviton modes in a thin band of mass up to order $m \leqslant \sqrt{2} \alpha$, including the massless graviton mode (expected on account of Lorentz covariance on the observable brane world). We associate the scale $\alpha$ with that of supersymmetry breaking on the 4-brane, as a result of the recoil process [28], and demonstrate that the corrections to the four-dimensional Newton's law are suppressed by powers of $\alpha / M_{s}^{2}$, with $M_{s} \sim 10^{18} \mathrm{GeV}$ in our scenario. Conclusions and outlook are presented in Sec. V.

\section{WORLD-SHEET APPROACH TO D-BRANE/D-BRANE SCATTERING: A REVIEW}

We first review the world-sheet formalism based on logarithmic operators that was developed in a series of papers $[24,25,29,30]$, for the mathematical description of the recoil of a D-brane when struck by a closed-string state or by another D-brane. Logarithmic conformal field theory [27] lies on the border between finite conformal field theories and general renormalizable two-dimensional quantum field theories. It is the relevant tool $[24,25,29]$ for this problem, because the recoil process involves a change of state (transition) in the string background, and as such is not described by a conformal field theory. This change of state induced by the recoil process can be described as a change in the $\sigma$-model background, and as such is a nonequilibrium process. This is reflected $[29,25]$ in the logarithmic operator algebra itself.

As discussed in Refs. [24,29,25] in the case of D-brane string solitons, their recoil after interaction with a closedstring (graviton) state is characterized by a $\sigma$ model deformed by a pair of logarithmic operators [27]

$$
C_{\epsilon}^{I}=\epsilon \Theta_{\epsilon}\left(X^{I}\right), \quad D_{\epsilon}^{I}=X^{I} \Theta_{\epsilon}\left(X^{I}\right), \quad I \in\{0, \ldots, 3\}
$$

defined on the boundary $\partial \Sigma$ of the string world sheet. Here $X^{I}, I \in\{0, \ldots, 3\}$ obey Neumann boundary conditions on the string world sheet, and denote the brane coordinates, while $\Theta_{\epsilon}\left(X^{I}\right)$ is the regularized step function, to be defined below. The remaining $y^{i}, i \in\{4, \ldots, 9\}$ denote the transverse bulk directions.

In the case of D particles, which were examined in Refs. $[24,29,25]$, the index $I$ takes the value 0 only, in which case the operators (1) act as deformations of the conformal field theory on the world sheet. The operator $U_{i} \int_{\partial \Sigma} \partial_{n} X^{i} D_{\epsilon}$ describes the movement of the $\mathrm{D}$ brane induced by the scattering, where $U_{i}$ is its recoil velocity, and $Y_{i} \int_{\partial \Sigma} \partial_{n} X^{i} C_{\epsilon}$ describes quantum fluctuations in the initial position $Y_{i}$ of the D particle. It has been shown rigorously [25] that the logarithmic conformal algebra ensures energy-momentum conservation during the recoil process $U_{i}=l_{s} g_{s}\left(k_{i}^{1}+k_{i}^{2}\right)$, where $k^{1}\left(k^{2}\right)$ is the momentum of the propagating closed string state before (after) the recoil, and $g_{s}$ is the string coupling, which is assumed here to be weak enough to ensure that D-branes are very massive, with mass $M_{D}=1 /\left(l_{s} g_{s}\right)$, where $l_{s}$ is the string length.

In the case of $\mathrm{D} p$-branes, the pertinent deformations are slightly more complicated. As discussed in Ref. [24], the deformations are given by

$$
\sum_{I} g_{I i}^{D} \int_{\partial \Sigma} \partial_{n} X^{i} D_{\epsilon}^{I} \quad \text { and } \quad \sum_{I} g_{I i}^{C} \int_{\partial \Sigma} \partial_{n} X^{i} C_{\epsilon}^{I}
$$

The $0 i$ components of the two-index couplings $g_{I i}^{\alpha}, \alpha$ $\in\{C, D\}$ include the collective momenta and coordinates of the D-brane as in the D-particle case above, but now there are additional couplings $g_{I i}^{\alpha}, I \neq 0$, which describe the folding of the D-brane. Such a folding may be caused by scattering with another macroscopic object, namely, another 


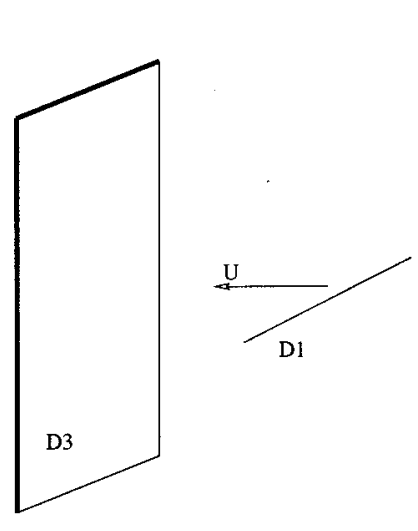

(a)

FIG. 1. Schematic representation of the folding effect in D-brane/D-brane collisions. (a) A D1 brane moving with velocity $U$ along a "bulk" direction perpendicular to a D3 brane embedded in a $D$-dimensional Euclidean space-time $E_{D}$ strikes the D3 brane (b), which is then folded, and the space-time around it is distorted into $\mathrm{AdS}_{3} \otimes E_{D-3}$. The dashed circle around the D1 direction in (b) indicates the angular deficit that appears when the bulk direction along which the D1 brane was moving is compactified to a circle. A generalization to a higher-dimensional case for the incident brane is straightforward. In that case the deficit (in the compact case) is a higher-dimensional solid hyperangle.

D-brane, propagating in a transverse direction, as shown schematically in Fig. 1 for the case of a D1-brane hitting a D3-brane. This situation is the most interesting to us, since it generates an $\mathrm{AdS}_{3}$ space, as we show below. For symmetry reasons, in the situation depicted in Fig. 1, the folding of the D3-brane occurs symmetrically around the axis of the D1brane. In this case, the precise logarithmic operator deformations shown in Eq. (2), which pertain only to the spatial region $y_{i}>0$ for the Dirichlet coordinates, should be supplemented with their counterparts for the $y_{i}<0$ region as well. This would, in principle, require additional $\Theta\left( \pm y_{i}\right)$ factors, which would complicate the analysis without introducing any new points of principle. Therefore, for simplicity, we restrict ourselves here to the $y_{i}>0$ patch of space-time, away from the hypersurface $y_{i}=0$. This will be implicit in what follows.

The correct specification of the logarithmic pair in Eq. (2) entails a regulating parameter $\epsilon \rightarrow 0^{+}$, which appears inside the $\Theta_{\epsilon}(t)$ operator

$$
\Theta_{\epsilon}\left(X^{I}\right)=\int \frac{d \omega}{2 \pi} \frac{1}{\omega-i \epsilon} e^{i \omega X^{I}}
$$

In order to realize the logarithmic algebra between the operators $C$ and $D$, one takes [24]

$$
\epsilon^{-2} \sim \ln [L / a] \equiv \Lambda,
$$

where $L(a)$ are infrared (ultraviolet) world-sheet cutoffs. The recoil operators (2) are relevant, in the sense of the renormalization group for the world-sheet field theory, having small conformal dimensions $\Delta_{\epsilon}=-\epsilon^{2} / 2$. Thus the $\sigma$ model perturbed by these operators is not conformal for $\epsilon$ $\neq 0$, and the theory requires Liouville dressing [31,32,29]. Momentum conservation is assured during the scattering process [25].

The folding couplings $g_{I i}^{D} \equiv g_{I i}, \quad I \in\{0, \ldots, p\}, \quad i \in\{p$ $+1, \ldots, 9\}$, are relevant couplings with world-sheet renormalization-group $\beta$ functions of the form

$$
\beta_{g_{I i}}=\frac{d}{d t} g_{I i}=-\frac{1}{2 t} g_{I i}, \quad t \sim \epsilon^{-2} .
$$

This implies that one may construct an exactly marginal set of couplings $\bar{g}_{I i}$ by redefining

$$
\bar{g}_{I i} \equiv \frac{g_{I i}}{\epsilon} .
$$

The renormalized couplings $\bar{g}_{0 i}$ were shown in Ref. [25] to play the role of the physical recoil velocity of the D-brane, while the remaining $\bar{g}_{I i}, I \neq 0$, describe the folding of the $\mathrm{D} p$-brane for $p \neq 0$. Here we shall assume, generalizing the case of Ref. [25] that the (bare) recoil couplings for all $I$ are of equal strength and related to the transverse momentum transfer as

$$
g_{I i}=g_{s} \frac{\Delta P_{i}}{M_{s}}, \quad I=0, \ldots, m, \quad i=m+1, \ldots, D
$$

for a D-brane embedded in a $D$-dimensional (bulk) spacetime.

A technical but important remark is now in order, concerning the world-sheet recoil formalism [24]. For reasons of convergence of the world-sheet path integral, the Neumann coordinate $X^{0}$ must be Euclideanized. It is only in this case that the identification (4), with $\epsilon^{2}>0$, leads to a mathematically consistent logarithmic algebra of operators. This can be understood simply by the fact that in the pertinent worldsheet computations of correlation functions of logarithmic operators (1) one encounters [24], due to Eq. (3), the free propagator of the Neumann coordinates $X^{I}$ :

$$
\mathcal{G}_{0}=\lim _{\sigma \rightarrow 0}\left\langle X^{I}(\sigma) X^{J}(0)\right\rangle_{*} \sim \eta_{I J} \ln [L / a],
$$

where $\langle\cdots\rangle_{*}$ denotes world-sheet partition function with respect to the free-string world-sheet action on a flat target space-time manifold $\left\{X^{I}\right\}$, and $\eta^{I J}$ is the target space metric. For Euclidean world sheets one takes $\eta^{I J}=\delta^{I J}$, and this is essential for the convergence of world-sheet path integral expressions entering in the respective correlators. Indeed, let us illustrate this by a simple example of the one-point function $\langle C\rangle$. This involves [see Eq. (3)] the computation of

$$
\begin{aligned}
& \left\langle\int_{-\infty}^{+\infty} \frac{d \omega}{\omega-i \epsilon} \exp \left(-i \omega X^{0}\right)\right\rangle_{*} \\
& \sim \int_{-\infty}^{+\infty} \frac{d \omega}{\omega-i \epsilon} \exp \left(-\frac{\omega^{2}}{2}\left\langle X^{0} X^{0}\right\rangle_{*}\right) .
\end{aligned}
$$


There are world-sheet ultraviolet infinities coming from the coincidence limit of the $X^{0}$ propagator in this expression, which after regularization give [24]

$$
\int_{-\infty}^{+\infty} \frac{d \omega}{\omega-i \epsilon} \exp \left(-\frac{\omega^{2}}{2} \eta^{00} \ln [L / a]\right) \text {. }
$$

Such integrals are convergent only for Euclidean $X^{0}$, which we have assumed in Ref. [24] and here.

The Euclideanization of the Neumann coordinates implies that in our picture, of viewing the $(3+1)$-dimensional world as a brane, the (longitudinal) Neumann coordinates will define a D4 domain wall in the bulk space-time, which, after analytic continuation of the coordinate $X^{0}$, will result in our four-dimensional space-time. However, the analytic continuation will take place only at the very end of the calculations. This will be very important for our purposes here, and will always be understood in what follows.

\section{RECOIL-INDUCED SPACE-TIME METRIC DEFORMATIONS}

As discussed in Refs. [29,26], the deformations (1) create a local distortion of the space-time surrounding the recoiling folded D-brane, which may be determined using the method of Liouville dressing. In Refs. $[29,26]$ we concentrated on describing the resulting space-time in the case when a $D$-particle, embedded in a $D$-dimensional space-time, recoils after the scattering of a closed string off the $D$-particle defect. To leading order in the recoil velocity $u_{i}$ of the $D$-particle, the resulting space-time was found, for times $t$ $\gg 0$ long after the scattering event at $t=0$, to be equivalent to a Rindler wedge, with apparent "acceleration" $\epsilon u_{i}$ [26], where $\epsilon$ is defined above Eq. (4). For times $t<0$, the spacetime is flat Minkowski. ${ }^{1}$

This situation is easily generalized to $\mathrm{D} p$-branes [33]. The folding or recoil deformations of the $\mathrm{D} p$-brane (2) are relevant deformations, with anomalous dimension $-\epsilon^{2} / 2$, which disturbs the conformal invariance of the $\sigma$ model, and restoration of conformal invariance requires Liouville dressing [32]. To determine the effect of such dressing on the space-time geometry, it is essential to write [29] the boundary recoil deformations as bulk world-sheet deformations

$$
\int_{\partial \Sigma} \bar{g}_{I_{z}} x \Theta_{\epsilon}(x) \partial_{n} z=\int_{\Sigma} \partial_{\alpha}\left[\bar{g}_{I z} x \Theta \Theta_{\epsilon}(x) \partial^{\alpha} z\right]
$$

where the $\bar{g}_{I z}$ denote the renormalized folding or recoil couplings (6), in the sense discussed in Ref. [25]. As we have already mentioned, such couplings are marginal on a flat world sheet. The operators (9) are marginal also on a curved world sheet, provided [32] one dresses the (bulk) integrand

\footnotetext{
${ }^{1}$ There is hence a discontinuity at $t=0$, which leads to particle production and decoherence for a low-energy spectator field theory observer who performs local scattering experiments long after the scattering, and far away from the location of the collision of the closed string with the $D$ particle [26].
}

by multiplying it by a factor $e^{\alpha_{I i} \phi}$, where $\phi$ is the Liouville field and $\alpha_{I i}$ is the gravitational conformal dimension, which is related to the flat-world-sheet anomalous dimension $-\epsilon^{2} / 2$ of the recoil operator, viewed as a bulk world-sheet deformation, as follows [32]:

$$
\alpha_{I i}=-\frac{Q_{b}}{2}+\sqrt{\frac{Q_{b}^{2}}{4}+\frac{\epsilon^{2}}{2}}
$$

where $Q_{b}$ is the central-charge deficit of the bulk world-sheet theory. In the recoil problem at hand, as discussed in Ref. [26],

$$
Q_{b}^{2} \sim \epsilon^{4} / g_{s}^{2}>0
$$

for weak folding deformations $g_{I i}$, and hence one is confronted with a supercritical Liouville theory. This implies a Minkowskian signature Liouville-field kinetic term in the respective $\sigma$ model [34], which prompts one to interpret the Liouville field as a timelike target-space field. However, in our context, this will be a second time coordinate [35], which is independent of the (Euclideanized) $X^{0}$. The presence of this second "time" for us will not affect the physical observables, which will be defined for appropriate slices of fixed Liouville coordinate, e.g., $\phi \rightarrow \infty$, or equivalently $\epsilon \rightarrow 0$. From the expression (11) we conclude [cf. Eq. (10)] that $\alpha_{I i} \sim \epsilon$ to leading order in perturbation theory in $\epsilon$, to which we restrict ourselves here.

We next remark that, as the analysis of Ref. [29] indicates, the $X^{I}$-dependent field operators $\Theta_{\epsilon}\left(X^{I}\right)$ scale as follows with $\epsilon: \Theta_{\epsilon}\left(X^{I}\right) \sim e^{-\epsilon X^{I}} \Theta\left(X^{I}\right)$, where $\Theta\left(X^{I}\right)$ is a Heavyside step function without any field content, evaluated in the limit $\epsilon \rightarrow 0^{+}$. The bulk deformations, therefore, yield the following $\sigma$-model terms:

$$
\frac{1}{4 \pi l_{s}^{2}} \int_{\Sigma} \sum_{I=0}^{3}\left(\epsilon^{2} \bar{g}_{I i}^{C}+\epsilon \bar{g}_{I i} X^{I}\right) e^{\epsilon\left(\phi_{(0)}-X_{(0)}^{I}\right)} \Theta\left(X_{(0)}^{I}\right) \partial_{\alpha} \phi \partial^{\alpha} y_{i},
$$

where the subscripts $(0)$ denote world-sheet zero modes, and $\bar{g}_{0 i}^{C}=y_{i}$.

Upon the interpretation of the Liouville zero mode $\phi_{(0)}$ as a (second) timelike coordinate, the deformations (12) yield space-time metric deformations (of the generalized spacetime with two times). The metric components for fixed Liouville-time slices can be interpreted in [29] as expressing the distortion of the space-time surrounding the recoiling D-brane soliton.

For clarity, we now drop the subscripts ( 0 ) for the rest of this paper, and we work in a region of space-time on the D3-brane such that $\epsilon\left(\phi-X^{I}\right)$ is finite in the limit $\epsilon \rightarrow 0^{+}$. The resulting space-time distortion is therefore described by the metric elements

$$
\begin{gathered}
G_{\phi \phi}=-1, \quad G_{i j}=\delta_{i j}, \quad G_{I J}=\delta_{I J}, \quad G_{i I}=0, \\
G_{\phi i}=\left(\epsilon^{2} \bar{g}_{I i}^{C}+\epsilon \bar{g}_{I i} X^{I}\right) \Theta\left(X^{I}\right), \quad i=4, \ldots, 9, \quad I=0, \ldots, 3,
\end{gathered}
$$


where the index $\phi$ denotes Liouville "time," not to be confused with the Euclideanized time which is one of the $X^{I}$. To leading order in $\epsilon \bar{g}_{I i}$, we may ignore the $\epsilon^{2} \bar{g}_{I i}^{C}$ term. The presence of $\Theta\left(X^{I}\right)$ functions and the fact that we are working in the region $y_{i}>0$ indicate that the induced space-time is piecewise continuous. ${ }^{2}$ In the general recoil or folding case considered in this article, the form of the resulting patch of the surrounding space-time can be determined fully if one computes the associated curvature tensors, along the lines of Ref. [26].

We now conclude this section with some remarks about the metric (13). First we restrict ourselves to the case of a single Dirichlet dimension $z$, playing the role of a bulk dimension in a setup where there are $X^{I}, I=0, \ldots, 3$ Neumann coordinates parametrizing a D4 (Euclidean) brane (our fourdimensional space-time). Upon performing the time transformation $\phi \rightarrow \phi-\frac{1}{2} \epsilon \bar{g}_{I z} X^{I} z$, the line element of the abovementioned space-time becomes

$$
\begin{aligned}
d s^{2}= & -d \phi^{2}+\left(\delta_{I J}-\frac{1}{4} \epsilon^{2} \bar{g}_{I z} \bar{g}_{J z} z^{2}\right) d X^{I} d X^{J} \\
& +\left(1+\frac{1}{4} \epsilon^{2} \bar{g}_{I z} \bar{g}_{J z} X^{I} X^{J}\right) d z^{2}-\epsilon \bar{g}_{I z} z d X^{I} d \phi,
\end{aligned}
$$

where $\phi$ is the Liouville field (which, we remind the reader, has Minkowskian signature, in the case of supercritical strings we are dealing with here).

One may now invoke a general coordinate transformation on the brane $X^{I}$ so as to diagonalize the pertinent inducedmetric elements in Eq. (14). ${ }^{3}$ For instance, to leading order in the deformation couplings $\bar{g}_{I z} \bar{g}_{J z}$, one may redefine the $X^{I}$ coordinates by

$$
\begin{aligned}
& X^{I} \rightarrow X^{I}-\frac{\epsilon^{2}}{8} z^{2} \bar{g}_{I z} \sum_{J \neq I} \bar{g}_{J z} X^{J}, \\
& z \rightarrow z\left(1+\frac{\epsilon^{2}}{8} \sum_{I \neq J} \bar{g}_{I z} \bar{g}_{J z} X^{I} X^{J}\right),
\end{aligned}
$$

which leaves only the diagonal elements of the metric tensor on the (redefined) hyperplane $X^{I}$. In that case, the metric becomes (to leading order in $g_{I z}^{2}$ ):

$$
\begin{gathered}
d s^{2}=-d \phi^{2}+\left(1-\alpha^{2} z^{2}\right)\left(d X^{I}\right)^{2} \\
+\left[1+\alpha^{2}\left(X^{I}\right)^{2}\right] d z^{2}-\epsilon \bar{g}_{I z} z d X^{I} d \phi, \\
\alpha=\frac{1}{2} \epsilon \bar{g}_{I z} \sim g_{s}\left|\Delta P_{z}\right| / M_{s},
\end{gathered}
$$

\footnotetext{
${ }^{2}$ The important implications for nonthermal particle production and decoherence for a spectator low-energy field theory in such space-times were discussed in Refs. [26,29], where only the $D$-particle recoil case was considered.

${ }^{3}$ Note that general coordinate invariance is assumed to be a good symmetry on the brane, away from the "boundary" $X^{I}=0$.
}

where in the last expression we wanted to make clear that, upon utilizing Eqs. (6),(7), one can actually express the parameter $\alpha$ (in the limit $\epsilon \rightarrow 0^{+}$) in terms of the (recoil) momentum transfer along the bulk direction. As we shall see later on, this parameter is responsible for the mass hierarchy in the problem, assuming that the string scale $M_{s}$ is close to Planck mass scale $10^{18} \mathrm{GeV}$, for ordinary string-theory couplings of order $g_{s}^{2} / 2 \pi=1 / 20$. The above metric element is derived in the case where $\epsilon \bar{g}_{I z} z \ll 1$.

A last comment concerns the case in which the metric (16) is exact, i.e., it holds to all orders in $\bar{g}_{I z} z$. This is the case where there is no world-sheet tree level momentum transfer. This naively corresponds to the case of static intersecting branes. However, the whole philosophy of recoil $[24,25]$ implies that even in that case there are quantum fluctuations induced by summing up genera on the world sheet. The latter implies the existence of a statistical distribution of logarithmic deformation couplings of Gaussian type about a mean field value $\bar{g}_{I z}^{c}=0$. Physically, the couplings $\bar{g}_{I z}$ represent recoil velocities of the intersecting branes, hence the situation of a Gaussian fluctuation about a zero mean value represents the effects of quantum fluctuations about the zero recoil velocity case, which may be considered as a quantum correction to the static intersecting brane case. Such Gaussian quantum fluctuations arise quite naturally by summing up higher world-sheet topologies [25]. We therefore consider taking a statistical average $\langle\langle\cdots\rangle\rangle$ of the line element (14),

$$
\begin{aligned}
\left\langle\left\langle d s^{2}\right\rangle\right\rangle= & -d \phi^{2}+\left(1-\frac{1}{4} \epsilon^{2}\left\langle\left\langle\bar{g}_{I z} \bar{g}_{J z}\right\rangle\right\rangle z^{2}\right) d X^{I} d X^{J} \\
& +\left(1+\frac{1}{4} \epsilon^{2}\left\langle\left\langle\bar{g}_{I z} \bar{g}_{J z}\right\rangle\right\rangle X^{I} X^{J}\right) d z^{2} \\
& -\epsilon\left\langle\left\langle\bar{g}_{I z}\right\rangle\right\rangle z d X^{I} d \phi,
\end{aligned}
$$

where

$$
\langle\langle\cdots\rangle\rangle=\int_{-\infty}^{+\infty} d \bar{g}_{I z}(\sqrt{\pi} \Gamma)^{-1} e^{-\bar{g}_{I z}^{2} / \Gamma^{2}}(\cdots),
$$

where the width $\Gamma$ has been calculated in Ref. [25], after proper summation over world-sheet genera, and in fact is found to be proportional to the string coupling $g_{s}$.

Obviously, from Eq. (18), and assuming that $g_{I z}=\left|U_{i}\right|$, where $U_{i}=g_{s} \Delta P_{i} / M_{s}$ is the recoil velocity [24,25], the average line element $d s^{2}$ becomes

$$
\begin{gathered}
\left\langle\left\langle d s^{2}\right\rangle\right\rangle=-d \phi^{2}+\left(1-\alpha^{2} z^{2}\right)\left(d X^{I}\right)^{2}+\left[1+\alpha^{2}\left(X^{I}\right)^{2}\right] d z^{2}, \\
\alpha=\frac{1}{2 \sqrt{2}} \epsilon \Gamma .
\end{gathered}
$$

The definition of $\alpha$ comes from evaluating the quantity $\left\langle\left\langle\bar{g}_{I z}^{2}\right\rangle\right\rangle$ using the statistical distribution (18). Thus, in that case, averaging over quantum fluctuations leads to a metric of the form (16), but with a parameter $\alpha$ much smaller, being determined by the width (uncertainty) of the pertinent quan- 
tum fluctuations [25]. The metric (19) is exact, in contrast to the metric (16) which was derived for $z \ll 1 / \alpha$. However, for our purposes below we shall treat both metrics as exact solutions of some string theory associated with recoil.

An important feature of the line element (19) is the existence of a horizon at $z=1 / \alpha$ for Euclidean Neumann coordinates $X^{I}$. Also notice that the Liouville field $\phi$ has decoupled, upon the averaging procedure, and this allows one to consider slices of this field, defined by $\phi=$ const, on which the physics of the observable world can be studied. From a world-sheet renormalization-group viewpoint this slicing procedure corresponds to selecting a specific point in the noncritical string theory space. Usually, the infrared fixed point $\phi \rightarrow \infty$ is selected. In that case, from Eq. (4), one considers a slice for which $\epsilon^{2} \rightarrow 0$. But any other choice could do, so $\alpha$ may be considered a small but otherwise arbitrary parameter of our effective theory.

The presence of a horizon raises the issue of how one could analytically continue so as to pass to the space beyond the horizon. The simplest way, compatible, as we shall show later with the low-energy Einstein's equations, is to take the absolute value of $1-\alpha^{2} z^{2}$ in the metric element (16). We therefore consider the following metric defined in all space $z \in R$ at a slice of the Liouville time $\phi=$ const:

$$
d s_{f}^{2}=\left|1-\alpha^{2} z^{2}\right|\left(d X^{I}\right)^{2}+\left[1+\alpha^{2}\left(X^{I}\right)^{2}\right] d z^{2} .
$$

For small $\alpha$, which is the case studied here, and for Euclidean Neumann coordinates $X^{I}$, the scale factor in front of the $d z^{2}$ term does not introduce any singular behavior, and hence for all qualitative purposes we may study the following metric element:

$$
d s_{f}^{2}=\left|1-\alpha^{2} z^{2}\right|\left(d X^{I}\right)^{2}+d z^{2},
$$

which is expected to share all the qualitative features of the full metric (20) induced by the recoil process in the case of an uncompactified "bulk" Dirichlet dimension $z$ we restrict ourselves here. ${ }^{4}$

A point that we would like to make concerns the fact that, formally, our analysis leading to Eq. (21) is valid in the region of bulk space-time for which $z>0$. However, one

${ }^{4}$ For the case of compact dimension $z$ the situation changes drastically, since in that case, for compact $z$ and at fixed $X^{I} \sim 1 / \epsilon \gg 0$ and $t \gg 0$, such that $\alpha^{2} X^{2} \simeq g_{X z}^{2} / 4$, we observe from the metric (20) that there exists a deficit angle in the circle around $z$ [33]:

$$
\delta \simeq\left(\pi g_{I z}^{2} / 4\right)
$$

implying the dynamical formation of a conical-like singularity. Such singularities in general break bulk space-time supersymmetry [28]. However, in view of the fact that the folded D-brane is an excited state of the string or D-brane system, the phenomenon should be viewed as a symmetry obstruction rather than a spontaneous breaking of symmetry, in the sense that, although the ground state of the string/D-brane system is supersymmetric, recoil produces a particular excited state that does not respect that symmetry [36]. We shall not, however, deal any further with the compact case in what follows, but instead assume a noncompact bulk dimension. may consider a mirror extension of the space-time for the region $z<0$, which we assume in this article. From now on, therefore, we treat the metric (21) as being defined over the entire real axis for the bulk coordinate $z \in R$. However, to make contact with the original recoil picture we restrict ourselves in regions of space-time for which $X^{I}>0$.

\section{A MASS HIERARCHY FROM RECOILING D-BRANES}

In this section we show that the metric obtained by the dynamical mechanism of D-brane scattering predicts a natural scale hierarchy. A crucial role is played by the value of the only parameter of the theory, i.e., $\alpha$, which is directly related to the D-brane recoil and appears in the warp factor in front of the four-dimensional part of the metric. With the above in mind we now write the metric (21) as

$$
\begin{aligned}
& d s_{f}^{2}=e^{-2 \sigma(z)}\left(d X^{I}\right)^{2}+d z^{2}, \\
& \sigma(z)=-\frac{1}{2} \ln \left(\left|1-\alpha^{2} z^{2}\right|\right) .
\end{aligned}
$$

The only nonzero components of the Christoffel symbol corresponding to the metric (23) read (in Euclidean signature for $\left.X^{I}, I=0, \ldots, 3\right)$

$$
\begin{gathered}
\Gamma_{04}^{0}=\Gamma_{40}^{0}=\sigma^{\prime}(z), \\
\Gamma_{00}^{4}=-\sigma^{\prime}(z) e^{-2 \sigma(z)}, \\
\Gamma_{i i}^{4}=\sigma^{\prime}(z) e^{-2 \sigma(z)}, \quad i=1,2,3, \\
\Gamma_{i 4}^{i}=\Gamma_{4 i}^{i}=-\sigma^{\prime}(z),
\end{gathered}
$$

where the prime denotes differentiation with respect to $z$. Notice that in the case of Minkowskian signature for the Neumann time coordinate $X^{0}$, the only change will be $\Gamma_{00}^{4}$ $\rightarrow-\Gamma_{00}^{4}$. This implies a similar sign change for the corresponding components of the Ricci curvature $R_{00} \rightarrow-R_{00}$. The curvature scalar therefore remains unchanged upon the analytic continuation of the time variable $X^{0}$.

For future use we note the following mathematical identities:

$$
\begin{aligned}
\sigma^{\prime}(z)= & -\frac{1}{2} \frac{\alpha}{|1+\alpha z|}[\Theta(1+\alpha z)-\Theta(-1-\alpha z)] \\
& +\frac{1}{2} \frac{\alpha}{|1-\alpha z|}[\Theta(1-\alpha z)-\Theta(-1+\alpha z)], \\
\sigma^{\prime \prime}(z)= & \frac{1}{2} \frac{\alpha^{2}}{(1+\alpha z)^{2}}[\Theta(1+\alpha z)-\Theta(-1-\alpha z)] \\
& +\frac{1}{2} \frac{\alpha^{2}}{(1-\alpha z)^{2}}[\Theta(1-\alpha z)-\Theta(-1+\alpha z)] \\
& -\frac{\alpha^{2}}{|1+\alpha z|} \delta(1+\alpha z)-\frac{\alpha^{2}}{|1-\alpha z|} \delta(1-\alpha z) .
\end{aligned}
$$

We next check on whether the metric (23) is a solution of Einstein's equations 


$$
\begin{gathered}
R_{\mu \nu}-\frac{1}{2} G_{\mu \nu} R=T_{\mu \nu}, \\
T_{\mu \nu}=-\frac{1}{4 M_{s}^{3}} G_{\mu \nu} \Lambda-\frac{1}{4 M_{s}^{3}} \sum_{i} \sqrt{G^{(i)}} G_{I J}^{(i)} \delta_{\mu}^{I} \delta_{\nu}^{J} \\
\times V_{(i)}(z) / \sqrt{G}, \quad \mu, \nu=0, \ldots, 4, I, J=0, \ldots, 3,
\end{gathered}
$$

where $M_{s}$ is the string mass scale and $\Lambda$ is a cosmological constant in the bulk space-time, and the sum $\Sigma_{i}$ is over possible D-brane defects. The index (i) denotes quantities pertaining strictly to such D-brane domain walls. In our case we can assume $i=1$, since originally we have a (struck) D-brane at the origin $z=0$. Note that, in a similar spirit to Ref. [8], we have subtracted a vacuum energy contribution, proportional to $V(z)$ from such D-brane defects.

It is easy to check from Eq. (25) that, by placing such domain walls at the horizon points $z= \pm 1 / \alpha$, one obtains that the metric (23) is indeed a solution of Eq. (26), provided that

$$
3 \sigma^{\prime \prime}(z)=\frac{1}{4 M_{s}^{3}} \sum_{i} V_{(i)}(z) \frac{\sqrt{G^{(i)}} G_{00}^{(i)}}{\sqrt{G} e^{-2 \sigma}}, \quad\left(\sigma^{\prime}\right)^{2}=-\frac{1}{24 M_{s}^{3}} \Lambda
$$

For a single D-brane at $z=0$, the solution is

$$
\begin{aligned}
\frac{\Lambda}{24 M_{s}^{3}}= & -\frac{1}{4} \frac{\alpha^{2}}{(1+\alpha z)^{2}}-\frac{1}{4} \frac{\alpha^{2}}{(1-\alpha z)^{2}}+\frac{1}{2} \frac{\alpha^{2}}{(1-\alpha z)^{2}} \mathcal{E} \\
\frac{V(z)}{4 M_{s}^{3}}= & \frac{3}{2} \frac{\alpha^{2}}{(1+\alpha z)^{2}}+\frac{3}{2} \frac{\alpha^{2}}{(1-\alpha z)^{2}} \\
& -\frac{3 \alpha^{2}}{|1+\alpha z|} \delta(1+\alpha z)-\frac{3 \alpha^{2}}{|1-\alpha z|} \delta(1-\alpha z),
\end{aligned}
$$

where $\mathcal{E}=[\Theta(1+\alpha z)-\Theta(-1-\alpha z)][\Theta(1-\alpha z)-\Theta(-1$ $+\alpha z)]=+1$, if $-1 / \alpha<z<1 / \alpha$, and $\mathcal{E}=-1$ otherwise.

The negative cosmological constant (anti-de Sitter type Universe) is a generic feature of intersecting branes [8], but also of the recoil formalism [35,33], and signals compatibility with space-time supersymmetry in the case of static nonrecoiling intersecting branes [8]. Notice, however, that the cosmological constant vanishes on the original brane $z=0$ and becomes infinitely negative on the horizon $|z|=1 / a$.

On the other hand, the vacuum energy distribution on the brane hypersurface, $V_{(z)}$, is positive $\left[V(0)=12 \alpha^{2}\right]$ at $z$ $=0$, which is compatible with the fact that the recoil excites the D-brane at $z=0$, and the excitation energy is of the same order as the kinetic energy transfer, due to energymomentum conservation [25]. $V_{(z)}$ also blows up negative at the horizon, signaling the formation of domain walls there.

Next we consider the issue of localization of bulk graviton states inside the horizon $-1 / \alpha<z<1 / \alpha$. To this end, we follow [8] and use the following ansatz for separating vari- ables $X^{I}$ and $z$, as far as (small) quantum fluctuations of the bulk graviton state $\hat{h}\left(X^{I}, z\right)$ about the background (23) are concerned

$$
\hat{h}\left(X^{I}, z\right)=\lambda(z) e^{i p_{I}^{E} X^{I}}
$$

where the notation $p_{I}^{E}$ in the momenta on the brane has been explicitly stated to remind the reader that we are working on a Euclidean set up for $\left\{X^{I}\right\}$, and hence for massive KK excitations, of mass squared $m^{2}>0$ the on-shell condition should read

$$
\left(p_{I}^{E}\right)^{2}=-m^{2}<0
$$

The equation for such small fluctuations can be obtained by linearizing Einstein's equations (26) around the AdS background and choosing appropriate gauge for the fluctuations of the metric. The final equation then reads

$$
\left[-\partial_{I} \partial^{I}-\partial_{z} \partial^{z}+\mathcal{V}(z)\right] \hat{h}\left(X^{I}, z\right)=0,
$$

where the "potential" $\mathcal{V}(z)$ arises from curvature.

Upon introducing the ansatz (28), and using Eq. (29), the above equation becomes a one-dimensional Schrödingertype eigenvalue equation for the bulk modes $\lambda(z)$ [8]

$$
-\lambda^{\prime \prime}(z)+\left[4\left(\sigma^{\prime}\right)^{2}-2 \sigma^{\prime \prime}\right] \lambda(z)=-m^{2} e^{2 \sigma} \lambda(z),
$$

where the various quantities are given in Eq. (25) for the problem at hand. It is important to note the minus sign in front of the mass term on the right-hand-side of Eq. (31), which is due to the Euclidean nature of $X^{I}$ hyperplane (29). As we shall see soon this will play an important physical role. Substituting Eq. (25) in Eq. (31) one obtains after some straightforward algebra:

$$
\begin{array}{r}
-\lambda^{\prime \prime}(z)-\left(\frac{2 \alpha^{2}}{\left|1-\alpha^{2} z^{2}\right|} \mathcal{E}-\frac{\alpha^{2}}{|1+\alpha z|} \delta(1+\alpha z)\right. \\
\left.\quad-\frac{\alpha^{2}}{|1-\alpha z|} \delta(1-\alpha z)+\frac{m^{2}}{\left|1-\alpha^{2} z^{2}\right|}\right) \lambda(z)=0, \\
\mathcal{E}=+1 \quad-1 / \alpha<z<1 / \alpha, \quad \mathcal{E}=-1 \quad \text { otherwise. }
\end{array}
$$

Equation (31) has the form of a one-dimensional Schrödinger equation. The potential is drawn in Fig. 2 for various values of the mass parameter $m$.

We observe the following. For mass parameters $0<m$ $<m_{c r} \equiv \sqrt{2} \alpha$ the potential is attractive, and the wave function peaks at $z=0$ (see Fig. 3). For mass parameter $m$ $>\sqrt{2} \alpha$ the wave functions are sharply peaked at the horizon $|z|=1 / \alpha$.

For illustration purposes, the wave functions (arbitrarily normalized) for three distinct cases, $m=0, m=0.2 m_{c r}$, and $m=0.8 m_{c r}$ are depicted in Fig. 3. For $m=0$ (massless graviton state), the corresponding wave function (solid curve) peaks at the D4-brane at $z=0$. As $m$ approaches the critical value $m_{c r}$ the wave fuction (dashed-dotted curve) spreads out 


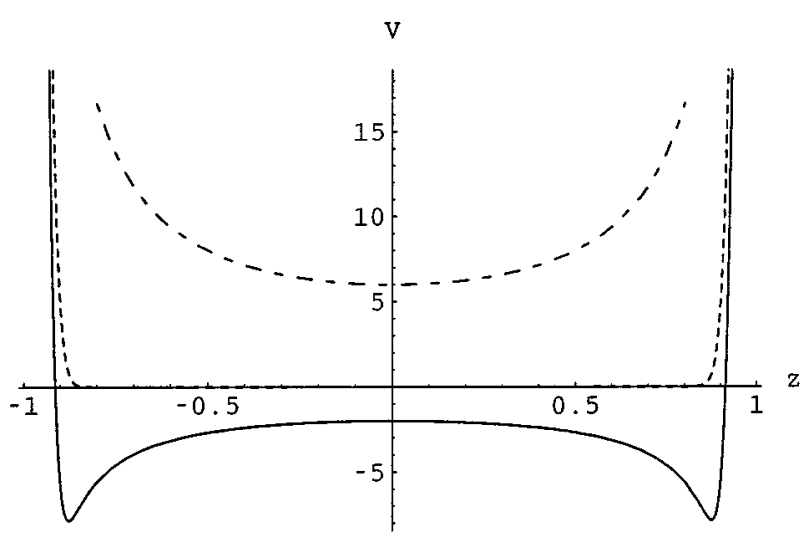

FIG. 2. Schematic representation of the potential of the equivalent Schrödinger equation in the bulk direction. The solid curve corresponds to the massless case while the dashed one represents the potential for the critical value $m=\sqrt{2} \alpha$. Beyond this value, the massive gravitons wave functions are no longer localized on the $z$ $=0$ D3-brane; the dashed-dotted curve represents the potential for such a case.

along the $z$ direction. Wave functions for $m>m_{c r}$ (shown in the figure with a dashed-dotted curve) are localized on the two boundaries $z= \pm 1 / \alpha$.

The spectrum is continuous in both cases and these are not bound states, in contrast to the case of Ref. [8] for the massless graviton mode. This is easily seen from the form of the corresponding zero energy eigenvalues in the Schrödinger equation (32). However, there is localization within the horizon of a thin band of Kaluza-Klein modes, with masses up to $\sqrt{2} \alpha$.

This leads to modifications of Newton's law on the brane hypersurface $\left\{X^{I}\right\}, I=0, \ldots, 3$ at $z=0$ (where the light band of modes including the massless one peaks, and where the cosmological constant vanishes). This can be easily calculated from the corresponding Green's function in the static potential between two mass sources $m_{1}, m_{2}$. The corrections

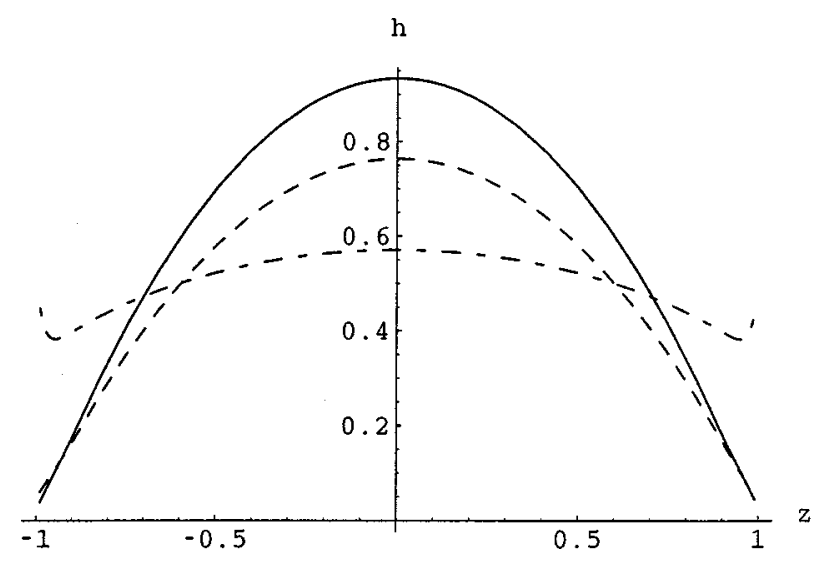

FIG. 3. The solution of the Schrödinger equation for three Kaluza-Klein modes with masses $0 \leqslant m<\sqrt{2} \alpha$. Solid curve corresponds to $m=0$ wave function. We observe that the modes are localized within the horizon $|z|<1 / \alpha$, while they start to spread as $m$ grows. For $m \geqslant \sqrt{2} \alpha$ they are peaked on the boundaries $\pm 1 / \alpha$. are generated by the exchange of massive modes with masses up to $\sqrt{2} \alpha$ :

$$
\begin{aligned}
V(r) & \sim G_{N} \frac{m_{1} m_{2}}{r}\left(1+\int_{0}^{\sqrt{2} \alpha} \frac{G_{N}}{M_{s}^{2}} m e^{-m r} d m\right) \\
& =G_{N} \frac{m_{1} m_{2}}{r}+\frac{G_{N}}{r^{3} M_{s}^{2}}\left(1-e^{-\sqrt{2} \alpha r}\right) m_{1} m_{2} \\
& \simeq G_{N} \frac{m_{1} m_{2}}{r}+\frac{G_{N} \sqrt{2} \alpha}{r^{2} M_{s}^{2}} m_{1} m_{2}
\end{aligned}
$$

for small thickness of the band $\alpha$. Thus, we see that the presence of massive Kaluza-Klein modes in the space-time (23), due to the presence of a recoiling brane (our world), struck by another string soliton, results in attractive corrections to Newton's law of $r^{-2}$ scaling, which are suppressed by a power of $\alpha / M_{s}$. In our case above the string scale may be assumed to be the conventional one, unlike the models of Refs. [5,7], $M_{s} \sim 10^{18} \mathrm{GeV}$. In the (formal) limit $\alpha \rightarrow \infty$ one recovers the situation discussed in [8], but as we have explained above our logarithmic formalism is valid for small $\alpha$.

The existence of thin bands of Kaluza-Klein modes sets a new mass hierarchy, in the sense that masses of order $\alpha$ may determine the supersymmetry-breaking scale on the brane $X^{I}$, due to the recoil process, as discussed in Refs. [28], where we refer the reader for details. Here we simply mention that the fact that the vacuum energy $V_{(z=0)}$ on the initial brane located at $z=0$ is positive and of order $12 \alpha^{2}$ [cf. Eq. (27)] signals supersymmetry breaking on the brane at a scale determined by $\alpha \ll M_{s}$, which may be taken to be of $\mathrm{TeV}$ scale, according to standard arguments on the gauge hierarchy problem of quadratic divergencies in four-dimensional spontaneously broken gauge theories. Notice that our picture for the hierarchy is different from that of Ref. [8], where the low mass scale (compared to $M_{s}$ ) on our world arises because of the small overlap of the bulk graviton wave function with that on the brane. In our picture, such small scale factors are valid only on the horizon at $|z|=1 / \alpha$, which, however, lies far away from the observable world.

The above considerations concern one noncompact bulk direction. The issue of compact bulk directions is complicated in our case because of the form of the metric (14), which implies deficits [cf. Eq. (22)], as discussed in Ref. [33]. It will be left for a future publication. Nevertheless, we believe that the results presented here are sufficient to demonstrate the important role of recoil (and in general quantum fluctuations) on the physics of large extra dimensions in string theory. The formation of bands of Kaluza-Klein modes, localized within the horizon of the metric (23) occurs here without the necessity of considering periodic lattice of branes. And actually the localization is obtained in a dynamical way, consistent with conformal field theory on the world sheet of the underlying string theory.

\section{CONCLUSIONS}

In this paper, we have made an attempt to generate dynamically the $M_{\text {Planck }}-M_{W}$ scale hierarchy in the context of 
D-brane scattering. Assuming a D4-brane embedded in a five-dimensional space-time we showed that scattering with another D-brane generates a bulk AdS5 space-time. The original D4-brane located at the origin of the fifth dimension is interpeted as our Euclideanized four-dimensional spacetime, where the cosmological constant is found to be zero. Taking into account deformations due to incorporated recoil effects, we calculated the space-time metric and showed that it satisfies the classical Einstein equations. Solving the linearized equation for the graviton modes, we find that there appears a band of massive lower Kaluza-Klein excitations, including the massless ordinary graviton state, which is localized in a small region of the fifth dimension around the origin where the D4-brane is located.

More precisely, due to the recoil quantum fluctuations of the D4-brane there is localization of a (continuous) thin band of massive KK states with masses up to $\sqrt{2} \alpha$, for small $\alpha$ $\ll M_{s}$, where the parameter $\alpha$ is related to the strength of the quantum fluctuations and sets the supersymmetry-breaking scale on the D4-brane. In this sense, the above approach generates dynamically a mass hierarchy, given the smallness of $\alpha$ as compared to the string scale $M_{s}$, which in our approach is assumed of order $10^{18} \mathrm{GeV}$. There is also the appearance of a horizon located at $|z| \sim 1 / \alpha$.

At present, our considerations pertain to noncompact fifth dimension. In the case of compact bulk dimensions there is a discrete set of allowed KK states, with masses quantized in units of the radius of the compact dimension. However, in that case, within the context of the recoil approach, there are induced deficits [33] which complicate the analysis. Such issues, together with the extension of the above approach to include more than one extra bulk dimension, are left for future work. However, we believe that the results presented here are of sufficient interest to motivate further studies along this direction.

\section{ACKNOWLEDGMENTS}

The work of N.E.M. is partially supported by P.P.A.R.C. (U.K.)
[1] A. Sen, Invited talk to be published in the proceedings of the 29th International Conference on High-Energy Physics (ICHEP 98), Vancouver, Canada, 1998, hep-ph/9810356.

[2] J. Polchinski, Phys. Rev. Lett. 75, 4724 (1995).

[3] V. A. Rubakov and M. E. Shaposhnikov, Phys. Lett. 125B, 136 (1983); 125B, 136 (1986).

[4] I. Antoniadis, Phys. Lett. B 246, 377 (1990).

[5] N. Arkani-Hamed, S. Dimopoulos, and G. Dvali, Phys. Lett. B 429, 263 (1998).

[6] I. Antoniadis, N. Arkani-Hamed, S. Dimopoulos, and G. Dvali, Phys. Lett. B 436, 257 (1998).

[7] I. Antoniadis and B. Pioline, Nucl. Phys. B550, 41 (1999).

[8] L. Randall and R. Sundrum, Phys. Rev. Lett. 83, 3370 (1999); 83, 4690 (1999).

[9] A. Kehagias and C. Sfetsos, hep-ph/9905417.

[10] E. G. Floratos and G. K. Leontaris, Phys. Lett. B 465, 95 (1999).

[11] J. Scherk, Phys. Lett. 88B, 265 (1979); K. Zachos, ibid. 76B, 329 (1978); T. Taylor and G. Veneziano, Phys. Lett. B 213, 450 (1988).

[12] S. Cullen and M. Pelerstein, Phys. Rev. Lett. 83, 268 (1999); L. Hall and D. Smith, Phys. Rev. D 60, 085008 (1999); V. Barger et al., Phys. Lett. B 461, 34 (1999).

[13] D. Krause and E. Fischbach, hep-ph/9912276.

[14] M. Visser, Phys. Lett. 159B, 22 (1985); M. Gogberashvili, hep-ph/9812296; Europhys. Lett. 49, 396 (2000).

[15] A. Kehagias, Phys. Lett. B 469, 123 (1999).

[16] W. D. Goldberger and M. B. Wise, Phys. Rev. D 60, 107505 (1999).

[17] J. Lykken and L. Randall, hep-th/9908076.

[18] C. Csaki, M. Graesser, C. Kolda, and J. Terning, Phys. Lett. B 462, 34 (1999); T. Nihei, ibid. 465, 81 (1999); N. Kaloper, hep-th/9912125.

[19] I. Oda, hep-th/9908104; Phys. Lett. B 472, 59 (2000).

[20] A. Brandhuber and K. Sfetsos, J. High Energy Phys. 10, 013 (1999); K. Behrndt and M. Cvetic, hep-th/9909058.
[21] H. Davoudiasl, J. L. Hewett, and T. G. Rizzo, Phys. Lett. B 473, 43 (2000); Phys. Rev. Lett. 84, 2080 (2000).

[22] P. Kanti, I. Kogan, K. Olive, and M. Pospelov, Phys. Lett. B 468, 31 (1999); P. Binetruy, C. Deffayet, U. Ellwanger, and D. Langlois, hep-th/9910219.

[23] H. Hatanaka et al., hep-th/9909076; S. Nam, hep-th/9911237; E. G. Floratos and G. K. Leontaris (unpublished).

[24] I. I. Kogan, N. E. Mavromatos, and J. F. Wheater, Phys. Lett. B 387, 483 (1996).

[25] N. E. Mavromatos and R. J. Szabo, Phys. Rev. D 59, 104018 (1999).

[26] J. Ellis, P. Kanti, N. E. Mavromatos, D. V. Nanopoulos, and E. Winstanley, Mod. Phys. Lett. A 13, 303 (1998).

[27] V. Gurarie, Nucl. Phys. B410, 535 (1993); J. S. Caux, I. I. Kogan, and A. M. Tsvelik, ibid. B466, 444 (1996); M. A. I. Flohr, Int. J. Mod. Phys. A 11, 4147 (1996); M. R. Gaberdiel and H. G. Kausch, Nucl. Phys. B489, 293 (1996); I. I. Kogan and N. E. Mavromatos, Phys. Lett. B 375, 111 (1996); M. R. Rahimi-Tabar, A. Aghamohammadi, and M. Khorrami, Nucl. Phys. B497, 555 (1997); I. I. Kogan, A. Lewis, and O. A. Soloviev, Int. J. Mod. Phys. A 13, 1345 (1998).

[28] A. Campbell-Smith and N. E. Mavromatos, Phys. Lett. B 476, 149 (2000).

[29] J. Ellis, N. E. Mavromatos, and D. V. Nanopoulos, Int. J. Mod. Phys. A 13, 1059 (1998).

[30] J. Ellis, N. E. Mavromatos, and D. V. Nanopoulos, Int. J. Mod. Phys. A 13, 5093 (1998).

[31] F. David, Mod. Phys. Lett. A 3, 1651 (1988).

[32] J. Distler and H. Kawai, Nucl. Phys. B321, 509 (1989).

[33] John Ellis, N. E. Mavromatos, and Elizabeth Winstanley, Phys. Lett. B 476, 165 (2000).

[34] I. Antoniadis, C. Bachas, J. Ellis, and D. V. Nanopoulos, Nucl. Phys. B328, 117 (1989).

[35] J. Ellis, N. E. Mavromatos, and D. V. Nanopoulos, Int. J. Mod. Phys. A 13, 5093 (1998).

[36] E. Witten, Int. J. Mod. Phys. A 10, 1247 (1995). 